\title{
Investigating the dielectric properties of barium titanate nanocomposites using transmission electron microscopy image processing
}

\author{
Gio Ferro ${ }^{1} \cdot$ Dithi Ganjam ${ }^{2}\left(\right.$ Maia Gibson $^{2} \cdot$ Katie Partington $^{2} \cdot$ Akshay Trikha $^{2} \cdot{\text { Mandy } \mathrm{Wu}^{2} \cdot \text { Jessica Domrzalski }}^{3}$. \\ Albert Dato ${ }^{2} \cdot$ Todd Monson $^{3}$ (i)
}

Received: 10 May 2021 / Accepted: 15 June 2021 / Published online: 14 July 2021

(c) The Author(s) 2021

\begin{abstract}
Barium titanate (BTO) is a ferroelectric perovskite material used in energy storage applications because of its high dielectric constant. A previous study showed that the dielectric constant for BTO nanoparticles drastically increases to over 15,000 at a particle size of $70 \mathrm{~nm}$. This result is highly contested, but its implications to energy storage motivated our investigation into the dielectric constants of BTO nanoparticles that are incorporated into a polymer matrix. We developed a novel method of using image processing techniques on transmission electron microscope images of BTO-polymer nanocomposites. Data on the positions, shapes, sizes, and orientations of BTO nanoparticles were used to build more realistic computational models that simulate the dielectric behavior of the nanocomposites. Here, we investigate the relationship between regions of enhanced electric field and the composite dielectric constant.
\end{abstract}

\section{Introduction}

Novel energy storage technologies aim to solve problems including the intermittent nature of some renewable energy sources, fast response demands of the electric grid, and electric vehicle charging. The advancement of electrostatic capacitors, which store energy in the form of an electric field, could help overcome such challenges. Since the discovery of the high dielectric constant of barium titanate (BTO), ceramics have been widely used as a dielectric material for capacitors [1-3]. Displacement of the titanium atom in BTO unit cells, visualized in Fig. S1, causes spontaneous polarization and ferroelectricity, which are the characteristics that are responsible for the high dielectric constant of BTO [4].

All the authors contributed equally to this work.

Todd Monson

tmonson@sandia.gov

1 Department of Chemistry, Pomona College, Claremont, CA 91711, USA

2 Department of Engineering, Harvey Mudd College, Claremont, CA 91711, USA

3 Sandia National Laboratories, Albuquerque, NM 87185, USA
In 2003, Wada et al. reported that BTO nanoparticle size greatly affects dielectric constant [5]. Specifically, powders with diameters $>300 \mathrm{~nm}$ were found to have a dielectric constant of 4000, whereas the dielectric constant surpassed 15,000 for a diameter of $70 \mathrm{~nm}$ [5]. These results indicate BTO may revolutionize energy storage, but are highly contested. Several other studies report BTO dielectric constants peaking at $\sim 5000-6000$ for particles with a $1000 \mathrm{~nm}$ diameter, then decreasing significantly with decreasing particle size $[1,6,7]$. Disagreement between studies into the effect of BTO particle size on dielectric constant motivates further research.

In 2018, Kaufman et al. investigated the dielectric constants of BTO nanoparticles using finite element analysis (FEA) [2]. All particles were assumed to be spherical and agglomerates were modeled as simple cubic arrangements of particles with zero separation between particles. Composites were built by embedding agglomerates in a polymer matrix, then adding randomly distributed particles around the cubic arrangements $[2,8]$. This approach was used to study various degrees of agglomeration in a controlled context to quantify effects of agglomeration on dielectric constant. Despite generating new knowledge, especially regarding enhanced field regions, the FEM developed by Kaufman et al. assumed cubic arrays of particles rather than real particle arrangements within the composite [2]. 
Here, we present a more realistic model of BTO nanocomposites by introducing ellipsoid particles of sizes, shapes, rotations, and arrangements directly obtained from transmission electron microscopy (TEM) images of fabricated nanocomposites. As depicted in Fig. 1, the Python programming language was used to extract particle information from TEM images and directly inform model geometry. COMSOL Multiphysics was used to build three-layer nanocomposites and calculate dielectric constants. Finally, the model was used to visualize how energy is stored within the composite. Regions of enhanced electric field (REEF), resembling those reported by Kaufman et al., were also observed.

\section{Materials and methods}

\section{Sample preparation and transmission electron microscopy}

$200 \mathrm{~nm}$ BTO particles were incorporated into epoxy composites of $20 \%$ volume loading (vol\%) as described in Section S3. The composite was cut along the edge around the copper plating on the surface and embedded in epoxy resin and then microtomed as described in Section S4. Finally, the slices were removed from water and mounted on a copper TEM grid using an inoculating loop. The resulting grid was imaged on a JEOL JEM 1200EX at different magnifications to achieve optimal resolution.

\section{Python image processing pipeline}

An image processing pipeline was created using Python's OpenCV computer vision library. Pre-processing and transformation steps detailed in Section S5.3 enable extraction of particle information. The pipeline centers around a watershed segmentation algorithm to obtain particle boundaries, as shown in Fig. S3 [9, 10].

Once particle contours are obtained, the longest and perpendicular short chord lengths are computed and particle rotations in the $X-Y$ plane are calculated. Since TEM images are two-dimensional, the third dimension is extrapolated. If both calculated radii are smaller than the actual radius of the imaged nanoparticles, or expected radius, the third radius is set as the expected radius. If any calculated radius is longer than the expected radius, the third radius is set as the average of the two calculated radii. Three TEM images are combined to form a layered model in which particles within a TEM image form a single layer of the composite, as shown in Fig. 1. The $z$-positions are randomized within layers.

Data are saved as a text file containing particle positions, radii, and rotations. Composite information including total particle numbers, BTO volume fraction, and electrode separation is also appended. Software was developed as described in Section S5.5 and the code repository for our project can be found in Section S1.
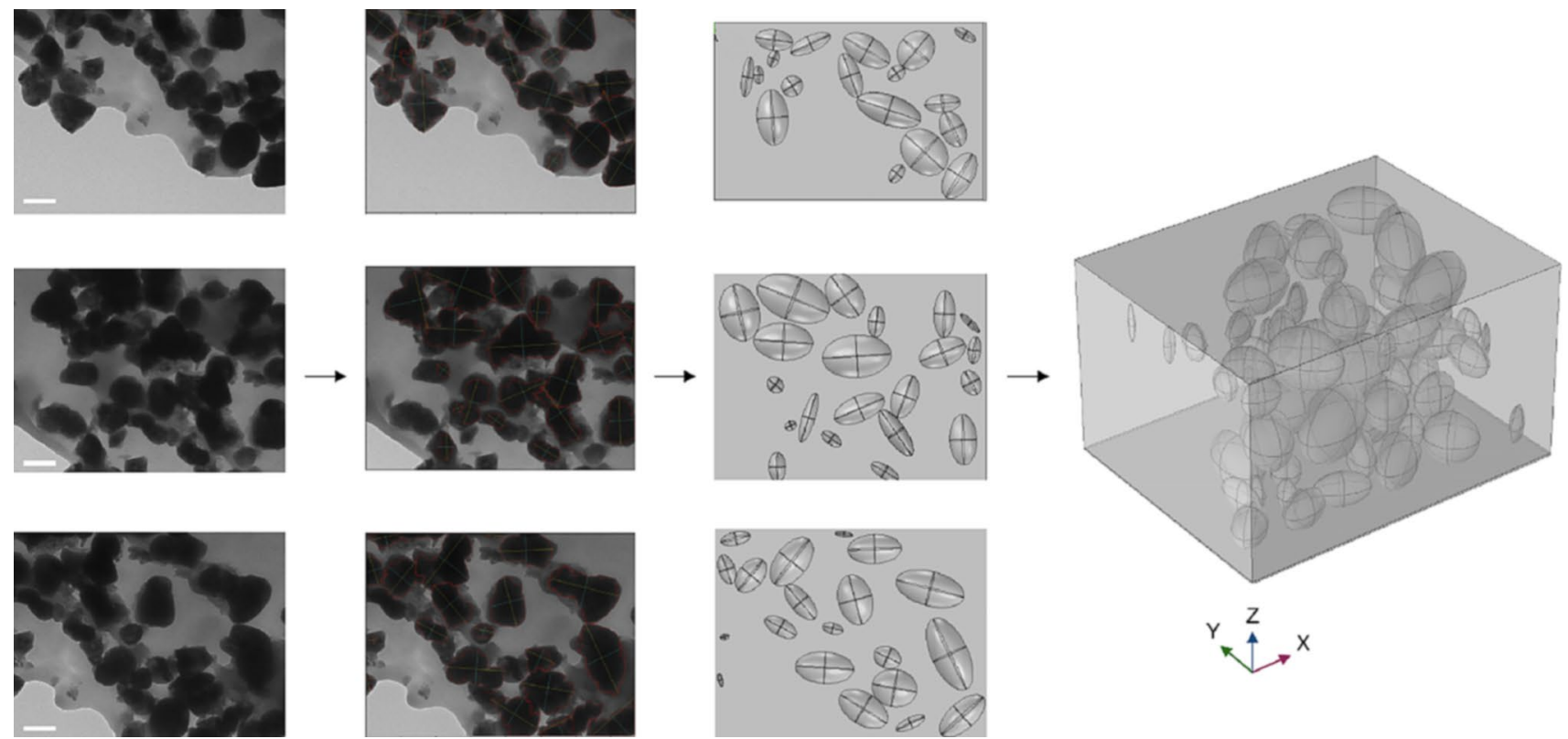

Fig. 1 Combining 3 TEM images of fabricated BTO nanocomposites embedded in an epoxy matrix into a three-layer COMSOL Mutliphysics model. Scale bar represents $200 \mathrm{~nm}$ 


\section{COMSOL multiphysics model}

COMSOL Multiphysics was used to model the nanocomposite from data obtained through the image processing pipeline. The model consists of a rectangular prism with copper electrodes on the top and bottom faces. Between these plates, ellipsoids representing BTO nanoparticles were suspended in a polymer matrix.

Data output by the Python pipeline was imported to COMSOL using MATLAB LiveLink. Once geometries were loaded, the matrix was set to epoxy. After meshing the model, an electrostatics study was run and surface integration across the copper plates was evaluated to calculate composite dielectric constant.

\section{Electric field visualizations}

Electric field visualizations were used to characterize particle interactions. The composite dielectric constant is calculated using internal energy and relates to electric field as shown in (1). Isosurface plots were generated by obtaining the magnitude of electric field from COMSOL's calculated values for electric potential. These visualizations provided insight on the locations of enhanced field regions.

The energy stored within any given region of the composite is

$U=\iiint \frac{1}{2} \varepsilon \varepsilon_{0} E^{2} \mathrm{~d} x \mathrm{~d} y \mathrm{~d} z$

where $\varepsilon_{0}$ is the vacuum permittivity, $E$ the electric field magnitude, and $\varepsilon$ the dielectric constant of the material at the point of integration.

\section{Results}

In producing a computational model of BTO nanocomposites in COMSOL, it is crucial to model REEF occurring between particles and between particles and electrodes. Equation (1) shows that the energy stored within any given region of the composite is proportional to the square of the electric field magnitude $E$. Therefore, increases in $E$ are expected to significantly impact $U$ and the composite dielectric constant $\varepsilon_{c}$ (see Section S6).

Kaufman et al. observed $E$ increasing when REEF were created by reducing distance between particles to the order of $1 \mathrm{~nm}$ in the applied field's direction. [2]. Kaufman et al. argued that the local electric field's magnitude can be increased by increasing agglomeration and decreasing distance between particles. The work done by Kaufman et al. modeled agglomerates as tightly packed spherical particles, but our model represents agglomeration using relative particle positions and rotations extracted from TEM images.

Quantifying the effects of REEF between particles involved exploring the relationship between layer spacing and $\varepsilon_{c}$. Figure 2a shows the relationship between the composite dielectric constant and distances between particles in the $z$-direction for a three-layered model derived from TEM images and a cubic array model, both at 20 vol\%. Model creation and the method of moving particles closer together is described in Section S7. Figure 2a shows that decreasing particle-particle distance in the direction of the electric field from $10 \mathrm{~nm}$ to $1 \times 10^{-13} \mathrm{~nm}$ causes $\varepsilon_{c}$ to increase in both the three-layer and cubic array models. The increase in dielectric constant reaches a plateau when the negative log of distance between particles is equal to 3 , or $1 \times 10^{-3} \mathrm{~nm}$.
Composite Dielectric Constant versus Particle-Particle Distance

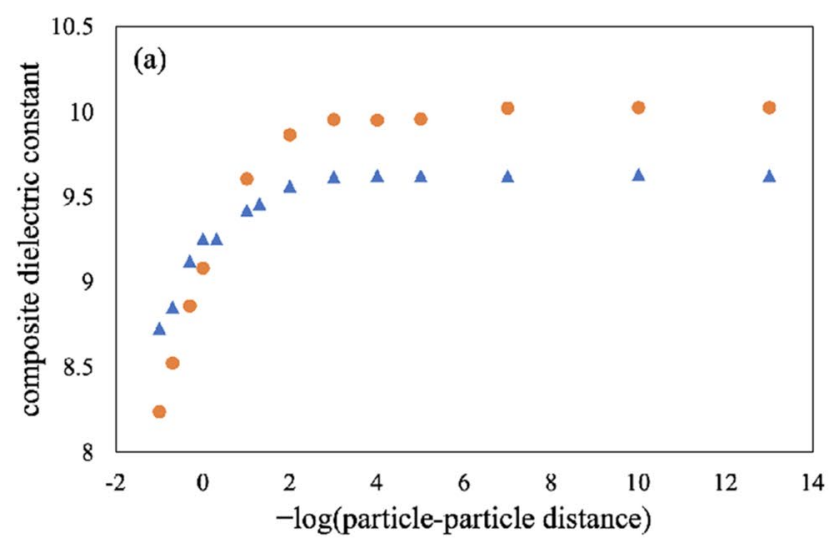

Composite Dielectric Constant versus Particle-Electrode Distance

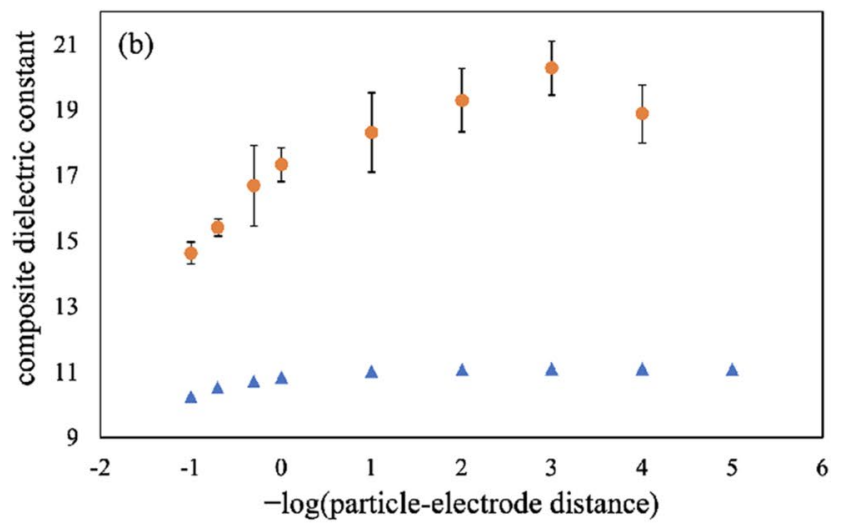

- Kaufman Cubic $\triangle$ TEM-obtained Three Layer Model

Fig. 2 Composite dielectric constant versus particle-particle distance (a) and particle-electrode distance (b) for two different models. For the TEM-obtained three-layer model, the $x$-values are upper bounds for the distances rather than exact values. Error bars are standard deviations 

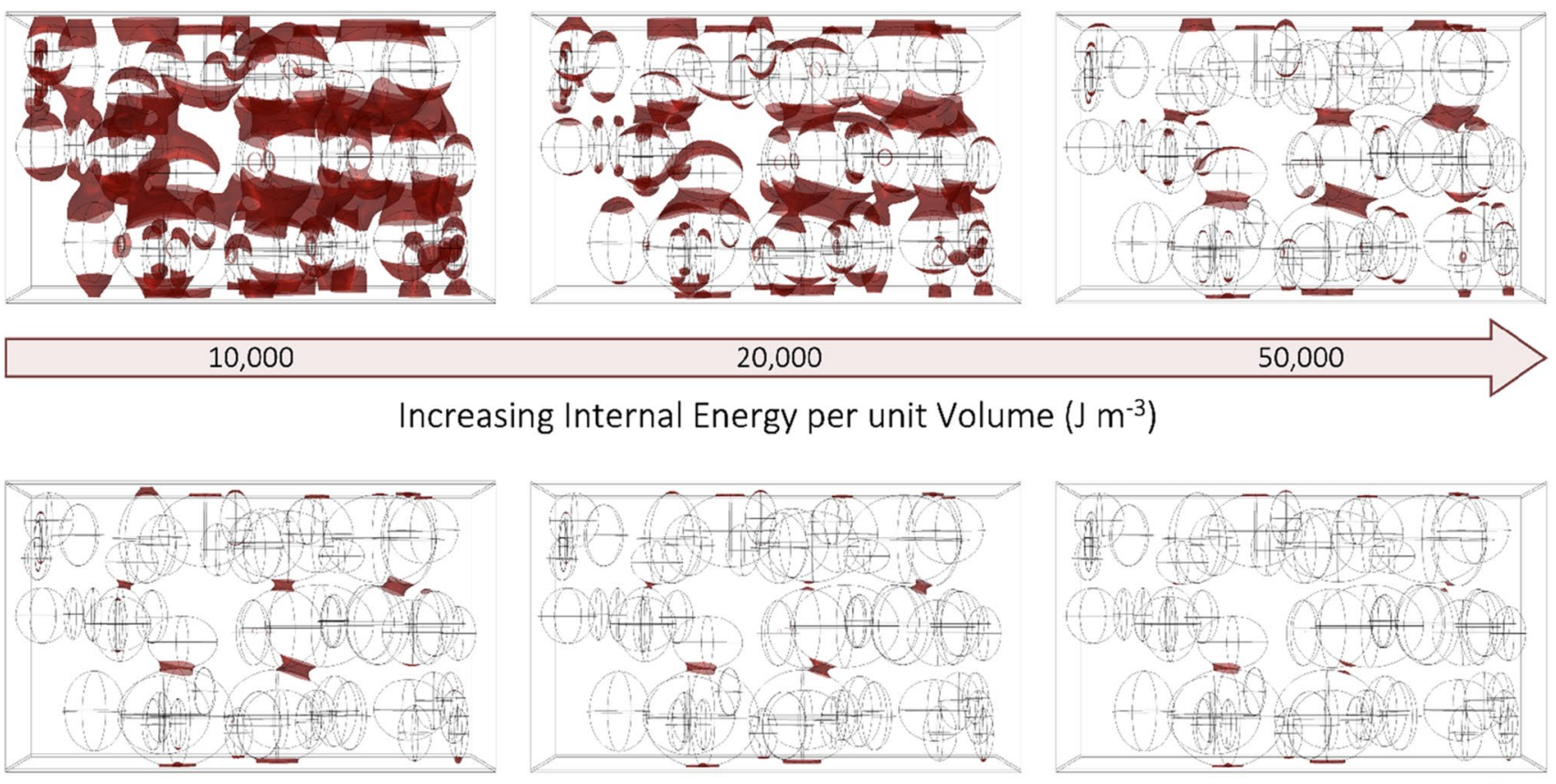

100,000

150,000

200,000

Increasing Internal Energy per unit Volume $\left(\mathrm{J} \mathrm{m}^{-3}\right)$

Fig. 3 Isosurfaces showing regions of high energy density within a TEM-obtained three-layer model. Only one energy density threshold value is shown in each plot and that value is constant along all the colored surfaces in that plot. As the energy density threshold

In the cubic array, particles are positioned directly above and below each other, increasing particle-particle electrostatic interactions between layers and thus $\varepsilon_{c}$.

Particle-electrode distances were decreased to further investigate the effects of REEF. Decreasing $z$-direction particle-electrode distance to less than $10 \mathrm{~nm}$ was found to increase $\varepsilon_{c}$. The three-layer model was used to generate Fig. 2a with a $z$-direction particle-particle distance of $1 \times 10^{-13} \mathrm{~nm}$ as a starting point. Next, the electrodes were incrementally pushed closer. Figure $2 \mathrm{~b}$ compares this data with that obtained when the same procedure was used on the cubic array model in Fig. 2a with $z$-distances between particles set to $0 \mathrm{~nm}$. Figure $2 \mathrm{~b}$ shows decreasing particleelectrode distance in the direction of the electric field that causes $\varepsilon_{c}$ to increase in the three-layer and cubic array models. There's a stronger correlation in the latter, likely from the larger number of REEF for particles equidistant from the electrodes. In the three-layer model, the effective spacing to capture interactions was found to be $0.0001 \mathrm{~nm}$. Decreasing particle-electrode distance further does not significantly impact $\varepsilon_{c}$ (notice the plateau in Fig. 2b).

Given the importance of REEF on $\varepsilon_{c}$, locations of these regions and the magnitude of internal energy stored were investigated using isosurface plots of internal energy per increases, there are fewer regions with such high internal energy per unit volume. These regions are usually between particles and between particles and electrodes

unit volume. These plots allow visualization of regions of the composite that meet a specific energy density threshold. Figure 3 shows the plots generated for a 13 vol\% model of $200 \mathrm{~nm}$ BTO in epoxy at various energy density thresholds. Notably, the composite has regions with an energy density as high as $200,000 \mathrm{Jm}^{-3}$. These regions of high energy density are mostly found between particles and the electrodes, but also exist where two particles are extremely close in the $\mathrm{z}$-axis or are oriented toward one another. At lower energy densities such as $10,000 \mathrm{Jm}^{-3}$, the volume of the composite meeting the threshold is far larger and comprised mainly of regions between close-packed particles, z-axis extremities of particles, and regions between particles and electrodes. REEF from particle-electrode proximity comprise less volume, but contain far higher energy densities.

REEF from relative particle orientation alongside particle-particle and particle-electrode separation are critical in constructing a model wherein effects of BTO nanoparticles on dielectric constant are captured. Effects of electrode positioning are presented in Section S8. While the model is still in development (see Section S9), use of ellipsoidal particles with parameters sourced from TEM images captures the importance of REEF and the effects of non-spherical BTO particle agglomeration on composite dielectric constant. 


\section{Conclusion}

The ability to model composites using TEM images has great promise in analyzing nanocomposites. The novel Python image processing pipeline enables the reconstruction of nanocomposites with geometric parameters of crosssection lengths, rotations, and relative particle placements sourced from experimental data. This pipeline may also be applied to analyze images from different applications containing agglomerated ellipsoids. Furthermore, the method of determining effective spacing between layers of particles as well as between the particles and the electrodes enables the modeling of REEF in three dimensions. This allows for in-depth physics-based analysis to be conducted on how the geometry of nanoparticles within a composite impacts dielectric constant. Computational modeling of nanocomposites using FEM presents great promise in developing new energy storage technologies and extracting the dielectric constant of discrete nanoparticles. The model's tunability to various TEM images lends itself to an understanding of the impact of the geometry of experimentally fabricated composites on energy storage capacity. Future work will focus on comparing composite dielectric constants from generated models to experimental data to understand what electromagnetic factors contribute to these values.

Supplementary Information The online version contains supplementary material available at https://doi.org/10.1557/s43580-021-00095-0.

Acknowledgments The authors wish to thank Dr. Susan Heidger of the Air Force Research Laboratory/High Power Microwave Electromagnetic Microwave Division for significant support of this work. Sandia National Laboratories is a multi-mission laboratory managed and operated by National Technology and Engineering Solutions of Sandia, LLC., a wholly owned subsidiary of Honeywell International, Inc., for the US Department of Energy's National Nuclear Security Administration under contract DE-NA-0003525. This paper describes objective technical results and analysis. Any subjective views or opinions that might be expressed in the paper do not necessarily represent the views of the US Department of Energy or the US Government.

Data availability The datasets generated during and/or analysed during the current study are available from the corresponding author on reasonable request.

\section{Declarations}

Conflict of interest The authors declare that they have no conflict of interest.

Open Access This article is licensed under a Creative Commons Attribution 4.0 International License, which permits use, sharing, adaptation, distribution and reproduction in any medium or format, as long as you give appropriate credit to the original author(s) and the source, provide a link to the Creative Commons licence, and indicate if changes were made. The images or other third party material in this article are included in the article's Creative Commons licence, unless indicated otherwise in a credit line to the material. If material is not included in the article's Creative Commons licence and your intended use is not permitted by statutory regulation or exceeds the permitted use, you will need to obtain permission directly from the copyright holder. To view a copy of this licence, visit http://creativecommons.org/licenses/by/4.0/.

\section{References}

1. G. Arlt, D. Hennings, G. de With, J. Appl. Phys. 58, 1619 (1985)

2. J. Kaufman, S. Tan, K. Lau, A. Shah, R. Gambee, C. Gage, L. MacIntosh, A. Dato, P. Saeta, R. Haskell, T. Monson, AIP Adv. (2018). https://doi.org/10.1063/1.5053442

3. P. Barber, S. Balasubramanian, Y. Anguchamy, S. Gong, A. Wibowo, H. Gao, H.J. Ploehn, H.-C. Zur Loye, Materials 2, 1697 (2009)

4. A. Messner, F. Eltes, P. Ma, S. Abel, B. Baeuerle, A. Josten, W. Heni, D. Caimi, J. Fompeyrine, J. Leuthold, J. Lightwave Technol. 37, 281 (2019)

5. S. Wada, H. Yasuno, T. Hoshina, S.-M. Nam, H. Kakemoto, T. Tsurumi, Key Eng. Mater. 301, 27 (2006)

6. M.H. Frey, Z. Xu, P. Han, D.A. Payne, Ferroelectrics 206/207, 337 (1998)

7. S.M. Aygün, J.F. Ihlefeld, W.J. Borland, J.-P. Maria, J. Appl. Phys. 109, 034108 (2011)

8. D. Brito, G. Quirarte, J. Morgan, E. Rackoff, M. Fernandez, D. Ganjam, A. Dato, T.C. Monson, MRS Commun. 10, 587 (2020)

9. S. Beucher, C. Lantuejoul, Proc. Int. Workshop Image Process. 2 , 1 (1979)

10. OpenCV (2020) 